\title{
Resistive wall wakefields in the extreme anomalous skin effect regime
}

\author{
Boris Podobedov* \\ National Synchrotron Light Source, Brookhaven National Laboratory, Upton, New York 11973, USA
}

(Received 2 January 2009; published 28 April 2009)

\begin{abstract}
Usual treatments of resistive wall effects in accelerators are limited to the normal skin effect regime of electrical conductivity in metals. Therefore they do not generally apply to the situations when beamexposed metallic surfaces of the vacuum chamber are held at cryogenic temperatures, where simple metals exhibit anomalous skin effect behavior. These situations occasionally occur in accelerators with cold-bore devices, such as small-gap superconducting undulators. The amount of anomalous resistivity material can be substantial to significantly influence beam dynamics. To accurately estimate these effects, we expand the conventional treatment of resistive wall in accelerators into the extreme anomalous skin effect region. Starting with the surface impedance expressions, we derive resistive wall related quantities commonly used in accelerator physics, such as wake functions, wake potentials, loss factor, etc. in the extreme anomalous skin effect region. We follow with examples for resistive wall generated heat and transverse mode-coupling instability.
\end{abstract}

DOI: 10.1103/PhysRevSTAB.12.044401

PACS numbers: 41.20.Jb, 41.60.- m, 41.75.-i

\section{INTRODUCTION}

Finite conductivity of accelerator vacuum chambers results in the interaction between the particle beam and its image currents flowing in the chamber walls. This interaction affects both longitudinal and transverse beam dynamics and its consequences are collectively known as "resistive wall effects," see e.g. [1-3]. For a uniform cylinder vacuum chamber geometry, the longitudinal effects scale inversely proportional to the pipe radius $b$, while transverse effects scale as $b^{-3}$. Thus, resistive wall becomes increasingly important for smaller apertures. Since the image currents penetration depth decreases with frequency (effectively increasing the impedance they encounter), the resistive wall effects become stronger for shorter bunches. These effects, such as beam energy loss, beam energy spread, transverse kick, currentdependent tune shift, and so on, have been studied in detail; however, the vacuum chamber material has been usually assumed to be in the regime of normal skin effect (NSE).

While the NSE regime commonly applies to accelerator components operating at room temperature, it generally does not hold when beam-exposed metal surfaces are at cryogenic temperatures. In the latter case, metals either become superconducting and resistive wall effects are typically weak [4] or, more commonly they enter the anomalous skin effect regime (ASE) where the AC conductivity of metals is substantially different from NSE.

To our knowledge, the resistive wall effect in the ASE regime has never been systematically studied, although its possibility has been mentioned in many papers. While cold bore superconducting magnets have been in use for a long time in proton machines, due to relatively large magnet

\footnotetext{
*boris@bnl.gov
}

apertures their resistive wall contribution to beam dynamics was negligible compared to that from the rest of the machine. Similarly, due to relatively long bunches, resistive wall heat fluxes were small in comparison to other sources, and thus could usually be ignored. One notable exception is the design of the LHC beam screen, for which resistive wall heating estimates were first done in Ref. [5]. Because of the importance of the subject for LHC, an experimental program was later established in CERN that confirmed the validity of ASE theory for this application (for frequencies up to $2 \mathrm{GHz}$ ) [6].

Over the past decade the situation has changed with the emergence of small-gap superconducting undulators in light sources [7-11]. A strong motivation behind these insertion devices (IDs) is that they can provide highly tunable hard x-ray photons at a relatively low electron beam energy, thus allowing for substantial cost savings. In superconducting undulators, periodic magnetic fields with periods on the order of $1 \mathrm{~cm}$ and magnitudes on the order of $1 \mathrm{~T}$ are created by superconducting coils. Beam image current path is provided by a thin layer usually made of $\mathrm{Cu}$, or $\mathrm{Cu}$-coated substrate. Magnetic design constraints place the image current layer into the cold part of a cryostat, i.e., these devices are cold bore. Most practical designs and operational devices work at $4 \mathrm{~K}$, a temperature where pure $\mathrm{Cu}$ is in the extreme ASE regime, to be discussed in detail later in the paper.

In a superconducting ID a relatively strong magnetic field with a short period necessitates small bore cross section, since the current carrying superconducting wires need to be as close to the beam as possible. User requirements for high photon brightness and flux argue for long undulators, and there is a general trend in modern light sources to install many undulators in the machine. Finally, electron bunches in modern light sources tend to be rela- 
tively short, at least in comparison to the proton machines. All of these factors strengthen the effects of the resistive wall, which, depending on the total length of superconducting undulators installed, could end up substantially influencing electron beam dynamics. Separately, in a superconducting undulator image current induced heat flux should be carefully accounted for, as it may substantially affect cryogenic design.

This is why we believe that detailed analysis of resistive wall effects in the ASE regime is in order. The purpose of this paper is therefore to systematically derive resistive wall related quantities in the regime of extreme ASE. The results of our analysis will apply to the resistive wall contributions due to superconducting undulators as well as to any other cold vacuum chamber components in the extreme ASE regime.

As stated above, this paper deals exclusively with the extreme ASE regime which happens only at cryogenic temperatures. We should mention that at short wavelengths (i.e. mm-range for pure copper) good conductors deviate from NSE and in fact may display anomalous resistivity behavior even at room temperature. In this case, a theoretical description of ASE is more complex as compared to the extreme ASE case, and the agreement with experiment is not always conclusive. Some recent accelerator-related references on the subject include $[12,13]$, while good reviews unrelated to accelerators are given in $[14,15]$.

We will limit ourselves to axially symmetric geometry and consider the most important $m=0$ wakefield pattern for the longitudinal and $m=1$ (dipole) pattern for the transverse. Only ultrarelativistic beams, $\gamma \gg 1$, will be considered, and we will limit the discussion to longitudinal distances $|z| \gg b / \gamma$. Gaussian units are used in the derivations throughout the paper. For convenience, we explicitly factor out the free space impedance $Z_{0}=4 \pi / c$ in the results, allowing for immediate conversion to MKS by replacing $Z_{0}$ by $120 \pi \Omega$.

The rest of the paper is organized as follows. In Sec. II we briefly review the skin effect regimes, introduce the surface impedance expression for the extreme ASE regime, and discuss its accuracy. In Sec. III we derive long-range wake functions and give expressions for the beam energy loss factor, beam energy spread, and the transverse kick factor. In Sec. IV we derive the short range wakefields and related quantities. Section V describes two applications of our results, specifically ohmic heating of cold bore undulators and resistive wall induced transverse mode-coupling instability.

\section{ANOMALOUS SKIN EFFECT}

Consider a linearly polarized plane EM wave $(E \propto$ $e^{-i \omega t}$ ) incident on a plane metal surface of conductivity $\sigma_{c}$. We assume that the metal is nonmagnetic and that it fills the half space $x>0$. The NSE regime holds when there is a local and instantaneous relation between the electric field and current density in the metal, i.e., one can write Ohm's law in the form

$$
\mathbf{J}=\sigma_{c} \mathbf{E}
$$

where $\sigma_{c}$ is a constant. In this case Maxwell's equations (neglecting the displacement current) result in the exponential field decay into the metal,

$$
E / E_{0}=H / H_{0}=J / J_{0}=\exp \left[-(1-i) x / \delta^{\mathrm{NSE}}\right],
$$

where the subscript denotes values at the surface, $\delta^{\mathrm{NSE}}$ is the classical skin depth,

$$
\delta^{\mathrm{NSE}}=\sqrt{\frac{2}{Z_{0} \sigma_{c} k}},
$$

and $k=\omega / c$ stands for the free space wave number. The ratio of tangential field components at the surface can be expressed through the Leontovich boundary condition, see e.g. [16],

$$
\mathbf{E}_{\mathbf{t}}=\zeta\left[\mathbf{H}_{\mathbf{t}} \times \mathbf{n}\right],
$$

where $\mathbf{n}$ is directed into the metal and $\zeta \equiv Z_{s} / Z_{0}$ denotes the surface impedance. For the normal skin effect regime the latter is defined by

$$
Z_{s}^{\mathrm{NSE}}(k)=\sqrt{\frac{k Z_{0}}{2 \sigma_{c}}}(1-i) .
$$

Here and below we give impedance expressions for $k \geq 0$. Since the fields must be real functions, $Z_{s}(-k)=Z_{s}(k)^{*}$.

The surface impedance given by Eq. (5) applies at sufficiently low frequencies and normal temperatures, where the assumption of temporarily and spatially constant conductivity in Eq. (1) holds true. At higher frequencies the inertia of electrons leads to relaxation effects, while their granularity manifests itself in ASE.

ASE is especially pronounced at low temperatures. Conductivity of pure metals increases several orders of magnitude when they are cooled from room temperature, where $\sigma_{c}$ is dominated by electron-phonon collisions, to LHe temperatures, where conductivity is impurity dominated, see e.g. [17]. A commonly used measure of purity is the residual resistivity ratio (RRR), defined (at $4 \mathrm{~K}$ ) as $\mathrm{RRR} \equiv \sigma_{c}(4 \mathrm{~K}) / \sigma_{c}(293 \mathrm{~K})$. RRR values in tens of thousands are achievable for ultrapure single crystal metal samples. More relevant for this paper is that bulk pieces of $\mathrm{Cu}$ and $\mathrm{Al}$ with $\mathrm{RRR}$ values of a hundred or more are commercially available for use in cryogenic components of accelerator vacuum chambers as well as other applications, see e.g. $[18,19]$.

By lowering the temperature of a reasonably high RRR metal, one could get to the regime where field penetration depth at a given frequency is shorter than the mean-free path of conducting electrons $l$. This is the ASE regime. Here only a small fraction of conducting electrons, with velocities directed at small angles to the surface, spend 
enough time in the field penetration layer to effectively contribute to AC conductivity. Therefore, for the same value of $\sigma_{c}$, AC conductivity is lower as compared to the NSE regime.

A detailed theory of anomalous resistivity for an isotropic normal conductor was first developed by Reuter and Sondheimer [20], see also [21-25]. Based on kinetic equations for the quasi-free-electron model, they obtained general expressions for the surface impedance in the form of fairly complicated integrals with complex parameters.

In the limit of extreme anomalous skin effect, defined by

$$
\begin{gathered}
\alpha=\frac{3}{2}\left(l / \delta^{\mathrm{NSE}}\right)^{2} \gg 1, \\
k \ll k_{p} v_{f} / c,
\end{gathered}
$$

where $k_{p} \equiv \omega_{p} / c$ is the wave number corresponding to the plasma frequency of conducting electrons, Reuter and Sondheimer obtained a simple expression for the surface impedance,

$$
Z_{s}(k)=B Z_{0}(1-\sqrt{3} i) k^{2 / 3},
$$

where

$$
B^{3}=\frac{\sqrt{3}}{16 \pi Z_{0}} l / \sigma_{c}
$$

The numerical coefficient in Eq. (9) corresponds to diffuse reflection of conducting electrons off the interface. A specular reflection assumption lowers $B$ by a factor of $8 / 9$ [20], thus the character of the surface interaction has only a minor effect in the extreme ASE regime. This no longer holds true for higher frequencies, when the condition of Eq. (7) is violated (and parameter $v_{f} / \omega$ overtakes $\delta$ in becoming the shortest length scale in the problem).

When parameter $\alpha$ of Eq. (6) is not too large, Eq. (8) loses accuracy, as compared to more complicated formulas obtained in [20] without restriction on $\alpha$, i.e., when ASE is not extreme. This is estimated in Appendix IV of [20], where surface impedance formulas are evaluated numerically at several values of $\alpha$. For instance, for diffuse reflection, for $\alpha=3$, the agreement is already well within a factor of 2, and for $\alpha=700$, the real and imaginary parts of the impedance exceed the extreme ASE values of Eq. (8) by $19 \%$ and $8 \%$, respectively. Chambers [22] has come up with approximate interpolating formulas to the results tabulated in [20] which could be useful to quickly estimate ASE surface impedance for a particular value of $\alpha$, and thus evaluate the accuracy of Eq. (8) for a particular application. Specifically, to account for finite $\alpha$, the real and imaginary parts of Eq. (8) are multiplied by the correction factor in the form $1+F \alpha^{-0.2757}$, where $F$ equals 1.157 and 0.457 for the real and imaginary parts, respectively, and $\alpha \geq 3$ is assumed [22].

For a given material and temperature, $\alpha$ is directly proportional to frequency. For instance, $\alpha=700$ corresponds to $1.4 \mathrm{GHz}$ (assuming $\mathrm{Cu}$ at $\mathrm{RRR}=100$ ) which is the characteristic frequency of a $34 \mathrm{~mm} \mathrm{rms}$ long Gaussian bunch. Since, for typical applications in electron accelerators, bunches are shorter (or even a lot shorter), corrections due to finite $\alpha$ are smaller than the percentage values quoted above. These corrections will be ignored through the rest of this paper.

Apart from $k^{2 / 3}$ frequency dependence, the most noteworthy feature of Eqs. (8) and (9) is that the conductivity $\sigma_{c}$ effectively cancels out since it enters $Z_{s}$ ratioed to the mean-free path. Indeed, according to the free-electron (Drude) model of metals, the conductivity is given by

$$
\sigma_{c}=\frac{n e^{2} l}{m v_{f}}=\frac{\omega_{p}^{2} l}{Z_{0} c v_{f}},
$$

where $n$ is the concentration of conducting electrons, $m$ is the effective mass, $\omega_{p}$ is the corresponding plasma frequency, and $v_{f}$ is the Fermi velocity. Since the concentration and hence $m v_{f} \sim \hbar n^{1 / 3}$ very weakly depend on temperature, the ratio $l / \sigma_{c} \propto v_{f} / n \propto n^{-4 / 3}$ is a temperature-independent material constant.

Therefore, while DC conductivity $\sigma_{c}(T)$ is a strong function of temperature $T$, the extreme ASE surface impedance and AC conductivity are temperature independent. An alternative way to put it is that extreme ASE surface impedance is independent of RRR. Note, however, that the lower frequency applicability limit for the extreme ASE regime, Eq. (6), strongly depends on RRR, $k_{\min } \propto$ $\mathrm{RRR}^{-3}$. This explains, for example, why alloys, that by design have a large amount of impurities and thus RRR values on the order of 1 , do not exhibit anomalous behavior even when cooled down to $\mathrm{LHe}$ temperatures.

For reference, we list in Table I the free-electron model parameters for $\mathrm{Al}$ and $\mathrm{Cu}$, which are the two most commonly used metals in accelerator cryogenics applications when high conductivity is required. Rewriting Eqs. (6) and (7) in terms of frequency, we find that for instance for $\mathrm{Cu}$ with $\operatorname{RRR}=100$, the applicability range of Eq. (8) is

TABLE I. Free-electron Fermi gas model parameters for $\mathrm{Al}$ and $\mathrm{Cu}$ [17] and constants $l / \sigma_{c}$ and $B$ calculated from Eqs. (9) and (10) [26].

\begin{tabular}{lccccc}
\hline \hline & $n 10^{28} \mathrm{~m}^{-3}$ & $v_{f} 10^{6} \mathrm{~m} / \mathrm{s}$ & $\omega_{p}{ }^{\mathrm{a}} 10^{16} \mathrm{rad} / \mathrm{s}$ & $l / \sigma_{c} 10^{-16} \Omega \mathrm{m}^{2}$ & $B 10^{-7} \mathrm{~m}^{2 / 3}$ \\
\hline $\mathrm{Al}$ & 18 & 2.0 & 2.4 & 4.0 & 3.3 \\
$\mathrm{Cu}$ & 8.5 & 1.6 & 1.7 & 6.6 & 3.9 \\
\hline \hline
\end{tabular}

${ }^{\mathrm{a}}$ Unscreened. 
$2 \mathrm{MHz} \ll f \ll 14 \mathrm{THz}$, while taking $\mathrm{RRR}=10$ shifts the lower limit to $2 \mathrm{GHz}$.

Assuming the same relation of the field penetration depth to the surface impedance as for the NSE regime, we see from Eq. (8) that the electric field penetrates the metal up to distances on the order of $\delta$ given by

$$
\delta^{3} \sim l\left(\delta^{\mathrm{NSE}}\right)^{2} .
$$

Note that, similar to the surface impedance, $\delta$ is independent of $\sigma_{c}$, as the latter enters only in combination $l / \sigma_{c}$. A detailed analysis of Ref. [20] confirms Eq. (11). On top of that it reveals that the field distribution in the ASE conductor is much more complex than in the NSE case. The distribution sensitively depends on the nature of electron scattering at the surface of the conductor; the distribution is generally nonexponential and there are tails that extend up to distances of order $l$.

When $l / \delta^{\mathrm{NSE}}>1$ but Eq. (6) does not hold, corrections to Eq. (8) in powers of $\delta^{\mathrm{NSE}} / l$ (or other simple functions of this parameter) have been obtained by analytical and/or empirical considerations, e.g. [22,24,27]. We could in principle expand the applicability range of the results to follow by including, at the expense of clarity, those correction terms. However, having in mind that our applications belong to the extreme ASE regime, we will not do so in this paper.

A derivation of Ref. [20] leading to the extreme ASE surface impedance expressions, Eqs. (8) and (9), did not assume an external magnetic field. Since we intend to apply our results to superconducting undulators which produce a DC magnetic field in the image current layer material, a comment on AC magnetoresistance is in order. Qualitatively, we expect the surface impedance corrections due to magnetic field to be small as long as the Larmor radius of conducting electrons is longer than $\delta$, i.e. $\delta$ remains the shortest length scale in the problem. For magnetic fields on the order of one Tesla, this condition is comfortably satisfied at $\mathrm{MHz}$ frequencies and higher, therefore we will ignore any effects of magnetic field below. Further discussion and experimental results for the magnitude of (very small) AC magnetoresistance in the ASE regime can found in $[28,29]$.

\section{BEAM IMPEDANCES AND WAKE FUNCTIONS}

First we derive the impedance in the anomalous skin effect regime by mostly following the normal skin effect regime derivation given in [1]. Consider an infinitely long smooth pipe with a round cross section of inner radius $b$. The walls are assumed thick enough so that no field leakage occurs to the outside. For the anomalous regime, it implies that $\delta$ should be much smaller than the wall thickness. We adopt a cylindrical coordinate system $s, r$, $\theta$. We consider an ultrarelativistic beam with charge density decomposed into multipole moments,

$$
\rho_{m}=\frac{I_{m}}{\pi a^{m+1}\left(1+\delta_{m 0}\right)} \delta(s-c t) \delta(r-a) \cos m \theta,
$$

where $I_{m}=q a^{m}, q$ is the total charge, $\delta(\cdots)$ denotes the $\delta$ function, and $\delta_{m 0}$ equals to 1 for $m=0$ and 0 otherwise.

Translational symmetry of the problem requires all fields depend on $z$ and $t$ only in combination $z=s-c t$, which we will take for the relative distance variable. Note that $z>0$ is ahead of the beam. We now write Maxwell's equation in cylindrical coordinates and then Fourier transform them with respect to $z$ using

$$
\begin{aligned}
& \left(E_{r}, E_{s}, H_{\theta}\right)=\cos m \theta \int \frac{d k}{2 \pi} e^{i k z}\left(\tilde{E}_{r}, \tilde{E}_{s}, \tilde{H}_{\theta}\right), \\
& \left(E_{\theta}, H_{r}, H_{s}\right)=\sin m \theta \int \frac{d k}{2 \pi} e^{i k z}\left(\tilde{E}_{\theta}, \tilde{H}_{r}, \tilde{H}_{s}\right) .
\end{aligned}
$$

\section{A. Longitudinal quantities}

Consider the $m=0$ case first. It immediately follows from Maxwell's equations in the metal-free region that the longitudinal component of electric field $\tilde{E}_{s}$ is independent of the transverse displacement for $r<b$. The rest of the field components can be expressed in terms of $\tilde{E}_{s}$ by

$$
\begin{gathered}
\tilde{E}_{r}=\tilde{H}_{\theta}=-i k \tilde{E}_{s} \frac{r}{2}, \quad r<a, \\
\tilde{E}_{r}=\tilde{H}_{\theta}=-i k \tilde{E}_{s} \frac{r}{2}+2 \frac{q}{r}, \quad a<r<b .
\end{gathered}
$$

It is now convenient to find the fields directly from the surface impedance, by making use of the Leontovich boundary condition at $r=b$,

$$
Z_{s}=-Z_{0} \tilde{E}_{s} / \tilde{H}_{\theta},
$$

where we assume the pipe radius to be much larger than the field penetration depth, i.e. $b \gg \delta$. This results in

$$
\tilde{E}_{s}(k)=-\frac{4 q / b}{2 Z_{0} / Z_{s}(k)-i k b} .
$$

At low frequencies, $k \ll(B b)^{-3 / 5}$, we can ignore the second term in the denominator. Then, from the definitions [1] of the longitudinal impedance and wake function given by

$$
\begin{gathered}
Z_{\|}(k)=\tilde{W}_{\|}^{\delta}(z)=\frac{1}{c} \int_{-\infty}^{\infty} e^{-i k z} W_{\|}^{\delta}(z) d z, \\
W_{\|}^{\delta}(z)=-\frac{1}{q} \int_{-L / 2}^{L / 2} E_{s}(z) d s
\end{gathered}
$$

we arrive to the expression

$$
Z_{\|}(k) / L=\frac{Z_{s}(k)}{2 \pi b} .
$$


Substituting Eq. (8) for the surface impedance above, we obtain the final expression for the extreme ASE longitudinal resistive wall impedance per unit length,

$$
Z_{\|}(k) / L=\frac{B Z_{0}}{2 \pi b}(1-\sqrt{3} i) k^{2 / 3} .
$$

We now proceed to derive the longitudinal wake function. Direct application of the inverse Fourier transform to Eq. (22) fails due to divergence at $k \rightarrow \infty$. This divergence is, however, not real but rather a trivial consequence of incorrect extrapolation of low frequency impedance to high frequencies. In reality, the high frequency longitudinal impedance goes to zero [see Eq. (41) of Sec. IV] which allows us to inverse Fourier transform Eq. (22) by integrating by parts and dropping the terms proportional to $Z_{\|}(\infty)$ [30]. This results in the longitudinal wake function:

$$
W_{\|}^{\delta}(z) / L=-\frac{2 B Z_{0} c}{3 b \pi|z|^{5 / 3} \Gamma\left(\frac{1}{3}\right)}, \quad z<0,
$$

where $\Gamma(\cdots)$ denotes the Gamma function, $\Gamma\left(\frac{1}{3}\right) \approx 2.68$. The wake function is negative, thus, similarly to the NSE case, the long-range wake accelerates a trailing charge, if it has the same sign as the leading charge $q$.

Clearly, $W_{\|}^{\delta}(z)$ differs from the usual wake function for the NSE regime, $W_{\|}^{\delta N S E} \propto \sigma_{c}^{-1 / 2}|z|^{-3 / 2}$, in two important aspects. First, as expected from Eq. (22), it scales differently with $z$. Second, as discussed in Sec. II, the material constant $B$ and, hence, the ASE wake function $W_{\|}^{\delta}(z)$ do not depend on conductivity $\sigma_{c}$.

Now consider a Gaussian bunch with the longitudinal distribution and its Fourier transform given by

$$
\begin{gathered}
\lambda(z)=\frac{1}{\sqrt{2 \pi} \sigma_{z}} \exp \left(-\frac{z^{2}}{2 \sigma_{z}^{2}}\right), \\
\tilde{\lambda}(k)=\exp \left(-\frac{k^{2} \sigma_{z}^{2}}{2}\right) .
\end{gathered}
$$

The total beam energy loss is conveniently expressed in terms of the loss factor, a quantity relating the energy change to the bunch charge by $\Delta \mathcal{E}=-k_{\text {loss }} q^{2}$. In the frequency domain, the loss factor can be calculated by

$$
k_{\text {loss }}=\frac{c}{\pi} \int_{0}^{\infty}|\tilde{\lambda}(k)|^{2} \operatorname{Re}\left[Z_{\|}(k)\right] d k
$$

It then follows from Eq. (22) that

$$
k_{\text {loss }} / L=\frac{B Z_{0} c \Gamma\left(\frac{5}{6}\right)}{4 b \pi^{2} \sigma_{z}^{5 / 3}} .
$$

The energy loss along the bunch is given by the longitudinal wake potential, which can be found from

$$
W_{\|}^{\sigma}(z)=\frac{c}{2 \pi} \int_{-\infty}^{\infty} e^{i k z} Z_{\|}(k) \tilde{\lambda}(k) d k .
$$

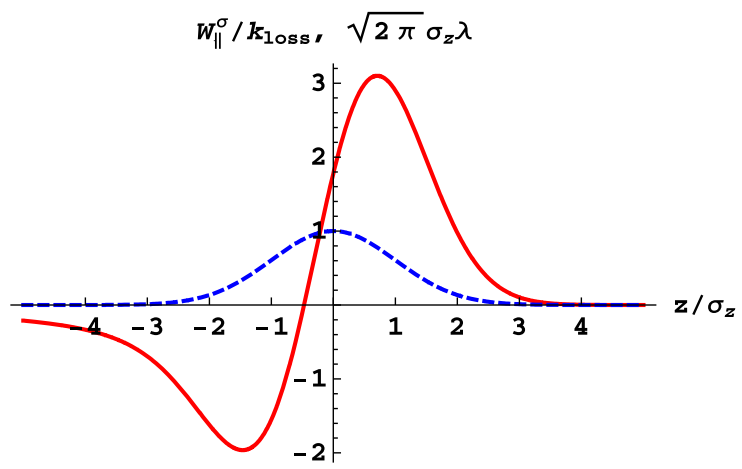

FIG. 1. (Color) Longitudinal wake potential (solid) and beam density (dashed); head of the bunch is $z>0 ; k_{\text {loss }}>0$ is given by Eq. (27).

For the Gaussian bunch it results in

$$
W_{\|}^{\sigma}(z) / L=-\frac{3 B Z_{0} c}{\sqrt{2} b \pi^{3 / 2} \sigma_{z}^{5 / 3} \Gamma\left(\frac{1}{3}\right)} G^{\prime}\left(z / \sigma_{z}\right),
$$

where

$$
G(x)=e^{-x^{2} / 2} \int_{0}^{\infty} e^{-\xi^{3}\left(\xi^{3} / 2+x\right)} d \xi,
$$

and the prime denotes the derivative with respect to the argument. The integral in Eq. (30) and hence the longitudinal wake potential above could also be expressed in terms of hypergeometric functions.

We plot this wake potential in Fig. 1. Particles towards the head of the bunch lose energy while particles in the tail experience energy gain. The wake potential looks similar to that of the NSE resistive wall, except, when normalized to $k_{\text {loss }}$, its extreme values are somewhat larger in magnitude. This could be traced to the $\sqrt{3}$ higher ratio of the imaginary part to the real part of the impedance in the ASE case.

The wake potential given by Eq. (29) is consistent with the loss factor found earlier, i.e.

$$
k_{\mathrm{loss}}=\int_{-\infty}^{\infty} \lambda(z) W_{\|}^{\sigma}(z) d z
$$

results in the loss factor given by Eq. (27).

Finally, since particles at different positions along the bunch lose (or gain) different amounts of energy, beam energy spread increases. From Eq. (29) we can calculate $q^{2} \sqrt{\int W_{\|}^{\sigma}(s)^{2} \lambda(s) d s-k_{\text {loss }}^{2}}$, and find the total wakeinduced rms energy spread,

$$
\sigma_{\mathcal{E}} \simeq 1.766 q^{2} k_{\text {loss }} .
$$

Note that the numerical coefficient in the right-hand side is appreciably higher than the one in the corresponding expression for the NSE resistive wall, $\sigma_{\mathcal{E}}^{\mathrm{NSE}} \simeq 1.056 q^{2} k_{\text {loss }}^{\mathrm{NSE}}$ [31]. 


\section{B. Transverse quantities}

We will now discuss $m=1$ transverse impedance and wake. Similar to the NSE resistive wall, it could be shown directly from Maxwell's equations that these quantities are related to their longitudinal $m=0$ counterparts by

$$
\begin{gathered}
Z_{\perp}(k)=\frac{2}{b^{2} k} Z_{\|}(k), \\
W_{\perp}^{\delta}(z)=\frac{2}{b^{2}} \int_{-\infty}^{z} W_{\|}^{\delta}(s) d s .
\end{gathered}
$$

Therefore we get the following expressions for the transverse impedance:

$$
Z_{\perp}(k) / L=\frac{B Z_{0}}{\pi b^{3}}(1-\sqrt{3} i) k^{-1 / 3}, \quad k>0,
$$

which can be extended to negative frequencies by $Z_{\perp}(-k)=-Z_{\perp}^{*}(k)$.

For the transverse wake function we obtain

$$
W_{\perp}^{\delta}(z) / L=\frac{2 B Z_{0} c}{b^{3} \pi|z|^{2 / 3} \Gamma\left(\frac{1}{3}\right)}, \quad z<0 .
$$

The transverse wake potential of a Gaussian bunch, $W_{\perp}^{\sigma}(z) \equiv \int \lambda\left(z^{\prime}\right) W_{\perp}^{\delta}\left(z-z^{\prime}\right) d z^{\prime}$, is given by

$$
W_{\perp}^{\sigma}(z) / L=\frac{3 \sqrt{2} B Z_{0} c}{b^{3} \pi^{3 / 2} \sigma_{z}^{2 / 3} \Gamma\left(\frac{1}{3}\right)} G\left(z / \sigma_{z}\right),
$$

where $G(\cdots)$ is the function introduced in Eq. (30).

Let us now discuss the kick factor, which is a key quantity characterizing the transverse single bunch dynamics [3]. For a bunch passing through the structure with bunch-centroid displacement $\left\langle r_{0}\right\rangle$, the kick factor, $k_{\text {kick}}$, defines the wake-induced bunch-centroid deflection $\left\langle r^{\prime}\right\rangle$ by

$$
\left\langle r^{\prime}\right\rangle=\frac{q}{\mathcal{E} / e} k_{\text {kick }}\left\langle r_{0}\right\rangle
$$

where $\mathcal{E}$ is the beam energy. In this definition, a positive kick factor corresponds to a defocusing force.

The kick factor could be expressed in terms of the transverse impedance or the wake potential by

$$
\begin{aligned}
k_{\mathrm{kick}} & =-\frac{c}{\pi} \int_{0}^{\infty}|\tilde{\lambda}(k)|^{2} \operatorname{Im}\left[Z_{\perp}(k)\right] d k \\
& =\int_{-\infty}^{\infty} \lambda(z) W_{\perp}^{\sigma}(z) d z .
\end{aligned}
$$

Using the equations in the beginning of this subsection, we find the final expression for the kick factor

$$
k_{\text {kick }} / L=\frac{B Z_{0} c}{b^{3} \pi \sigma_{z}^{2 / 3} \Gamma\left(\frac{2}{3}\right)} .
$$

Finally, in Fig. 2 we plot the transverse wake potential normalized to the kick factor. Unlike its longitudinal counterpart, the transverse wake potential does not switch the sign, i.e., all particles in the bunch experience defocusing

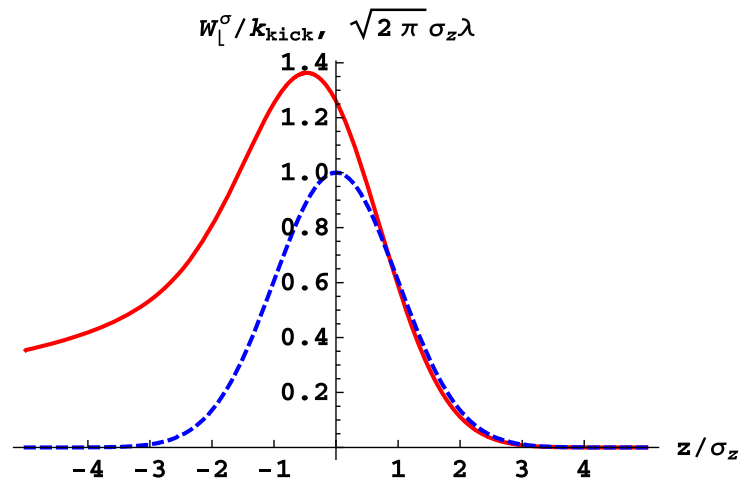

FIG. 2. (Color) Transverse wake potential (solid) and beam density (dashed); head of the bunch is $z>0 ; k_{\text {kick }}>0$ is given by Eq. (40).

force. It is the strongest at $z \simeq-0.471 \sigma_{z}$ where $W_{\perp}^{\sigma}(z) \simeq$ $1.363 k_{\text {kick }}$. When the wake potential is normalized as shown, it looks fairly similar to that of the NSE resistive wall.

\section{SHORT RANGE WAKES}

The results of the previous section, starting from Eq. (21), were derived assuming $k \ll(B b)^{-3 / 5}$. This implies the long-range wakes obtained above are correct down to distances on the order of $\left|z_{\min }\right| \sim(B b)^{3 / 5}$. The same scale limits the validity range of the bunch length dependence present in the wake potentials, loss factor, kick factor, etc.

If evaluated at shorter distances, the long-range expressions may lead to unphysical results. For instance, Eq. (23) gives negative values of $W_{\|}^{\delta}(z)$ for any $z<0$. This implies acceleration of a test particle that immediately follows the leading charge, which is clearly incorrect.

Assuming for a moment that the surface impedance given by Eq. (8) extends to infinite frequency, we will now derive the wake function and related quantities that should be correct down to arbitrarily short distances. We return to the expression for the longitudinal electric field, Eq. (18), and this time proceed by keeping the second term in the denominator. Rewriting Eq. (18) in terms of impedance, $Z_{\|} / L=-\tilde{E}_{s} /(q c)$, and plugging in Eq. (8) we get

$$
Z_{\|}(\bar{k}) / L=\frac{2 Z_{0}(B b)^{3 / 5}}{\pi b^{2}} \frac{\bar{k}^{2 / 3}}{1+i\left(\sqrt{3}-2 \bar{k}^{5 / 3}\right)},
$$

where we introduced dimensionless frequency $\bar{k} \equiv$ $k(B b)^{3 / 5}$.

Separating the real part and applying the cosine transform, we obtain the following expression for the wake function for $z<0$ :

$$
W_{\|}^{\delta}(z) / L=\frac{3 Z_{0} c}{5 \pi^{2} b^{2}} \int_{0}^{\infty} \frac{\cos \left(p^{3 / 5} \csc (\xi) z / s_{0}\right)}{p^{2}-\sqrt{3} p+1} d p,
$$

where we introduced a new length scale parameter $s_{0}$ by 


$$
s_{0} \equiv \csc (\xi)(B b)^{3 / 5}
$$

where $\xi \equiv \pi / 10$, and $\csc (\xi)=(1+\sqrt{5}) \simeq 3.23$. The choice of the numerical factor in Eq. (43) will be explained shortly.

In contrast to the long-range expression given by Eq. (23), the wake function above has correct asymptotic behavior. Specifically, for small values of $|z|$ the integral is positive, therefore the wake force directly behind the drive particle is repulsive. At $z \rightarrow-0$ Eq. (42) converges to $c Z_{0} / \pi b^{2}$. This value, independent of the surface impedance, can be obtained directly from energy conservation considerations [1]. At large values of $|z|$ Eq. (42) converges to Eq. (23), while at short distances the cosine function in the integral results in some oscillatory behavior.

To better illustrate the short range wake function behavior, we seek an alternative representation of Eq. (42). We analytically extend the impedance given by Eq. (41) to the complex $\bar{k}$ plane, and then perform the inverse Fourier transform to obtain the wake function. The integration is performed using standard techniques of complex calculus, see e.g. [32] for a similar derivation for the case of NSE resistive wall wake. Note that $Z_{\|}(\bar{k})$ has no singularities in the upper half plane, guaranteeing the causality of the wake function, $W_{\|}^{\delta}(z>0)=0$. For $z<0$ the inverse Fourier transform of Eq. (41) is calculated by closing the contour into the lower half plane, where the impedance function has two simple poles, $\bar{k}_{1}=\exp (-i \pi / 10)$ and $\bar{k}_{2}=$ $\exp (i 11 \pi / 10)$.

The wake function found in this manner naturally comes in two parts. The first term is a damped broadband resonator due to pole residues. The second contribution, which, by analogy with [32], we will call the diffusion term, comes from the contributions along the branch cut. The final expression is

$$
\begin{aligned}
W_{\|}^{\delta}(z) / L= & \frac{6 c Z_{0}}{5 b^{2} \pi}\left(e^{z / s_{0}} \cos \left[\cot (\xi) z / s_{0}\right]\right. \\
& \left.-\frac{\sqrt{3}}{4 \pi} \int_{0}^{\infty} \frac{e^{p^{3 / 5} \csc (\xi) z / s_{0}}}{p^{2}+p+1} d p\right), \quad z<0 .
\end{aligned}
$$

The resonator term has a wave number of $\cot (\xi) / s_{0}$, and the quality factor $Q=\frac{1}{2} \cot \xi \approx 1.5$. The diffusion term is a monotonic function of $z$. At short distances the resonator term dominates, i.e., it can be thought of as a short range wake. At long distances this term decays away and the diffusion term becomes the long-range wake function given by Eq. (23).

Our choice for the numerical coefficient in Eq. (43) that defines the characteristic distance $s_{0}$ for the ASE regime is now clear. It was made so that $s_{0}$ is exactly equal to the efolding distance for the resonator term. This definition is consistent with that of Bane and Sands [32], who introduced the characteristic distance for the NSE regime,

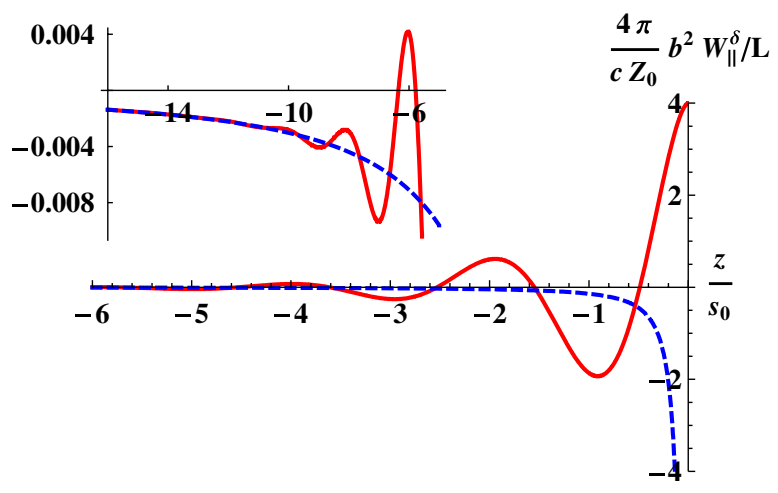

FIG. 3. (Color) Longitudinal wake function given by Eq. (44) (solid) and long-range approximation from Eq. (23) (dashed).

$$
s_{0}^{\mathrm{NSE}}=\left(\frac{2 b^{2}}{Z_{0} \sigma_{c}}\right)^{1 / 3}
$$

as the e-folding distance for the broadband resonator term of the longitudinal resistive wall wake function.

In Fig. 3 we plot the function given by Eq. (44) as well as its long-range counterpart, Eq. (23). The two curves converge closely at distances about $10 s_{0}$ behind the leading charge. At shorter distances, the wake function, increasingly dominated by the resonator term, exhibits a substantial amount of "ringing" and undergoes seven zero crossings. In comparison, the NSE short range resistive wall wake is more damped, $Q^{\mathrm{NSE}}=\sqrt{3} / 2$, and the wake function switches sign only 3 times [32].

We also obtain the expression for the loss factor in the form

$$
k_{\text {loss }} / L=\frac{3 c Z_{0}}{10 b^{2} \pi^{2}} \int_{0}^{\infty} \frac{e^{-\left[p^{3 / 5} \csc (\xi) \sigma_{z} / s_{0}\right]^{2}}}{p^{2}-\sqrt{3} p+1} d p .
$$

We plot it in Fig. 4 together with the long-range approximation found earlier (shown in dash). The loss factor no longer diverges for short bunches, in fact, for $\sigma_{z} / s_{0} \rightarrow 0$,

$$
k_{\mathrm{loss}} / L \rightarrow \frac{c Z_{0}}{2 b^{2} \pi}\left(1-\frac{3 \Gamma(1 / 6)}{5 \pi}\left[\csc (\xi) \sigma_{z} / s_{0}\right]^{5 / 3}\right) .
$$

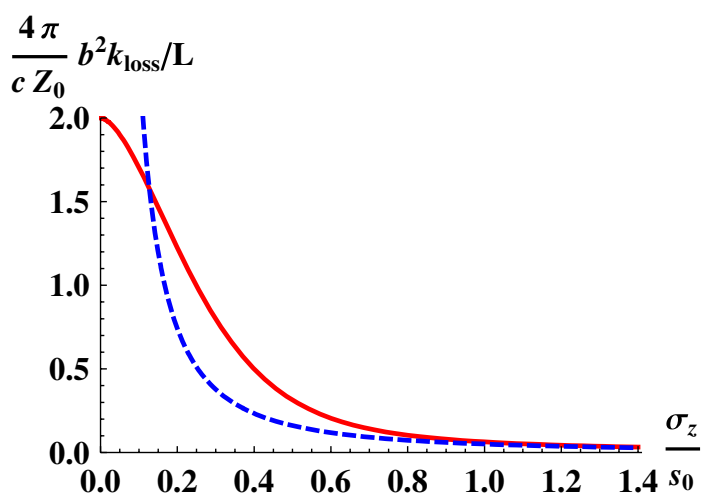

FIG. 4. (Color) Loss factor given by Eq. (46) (solid) and longrange approximation from Eq. (27) (dashed). 

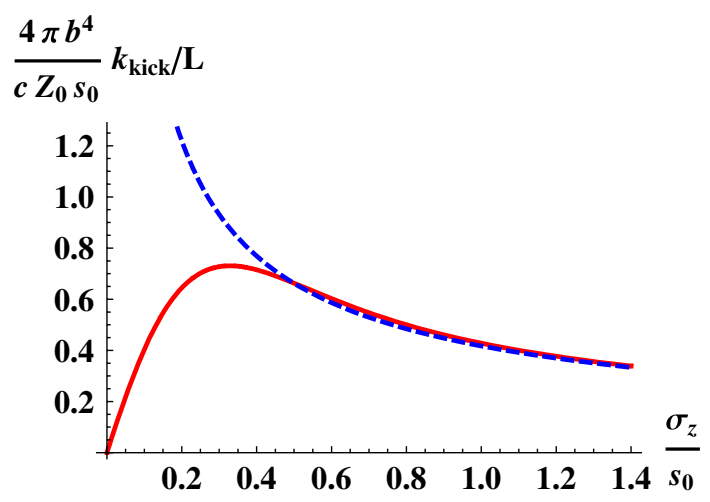

FIG. 5. (Color) Kick factor given by Eq. (48) (solid) and longrange approximation from Eq. (40) (dashed).

Also note that the long-range approximation for the loss factor is quite accurate for bunches longer than $s_{0}$.

Finally, the transverse functions can be obtained from the longitudinal using Eqs. (33) and (34). As an example we give the expression for the kick factor

$$
\begin{aligned}
k_{\text {kick }} / L= & \frac{3 c s_{0} Z_{0}}{5 b^{4} \pi^{2} \csc \xi} \\
& \times \int_{0}^{\infty} \frac{e^{-\left[p^{3 / 5} \csc (\xi) \sigma_{z} / s_{0}\right]^{2}}(\sqrt{3}-2 p)}{\left(p^{2}-\sqrt{3} p+1\right) p^{3 / 5}} d p,
\end{aligned}
$$

which we also plot in Fig. 5. Clearly the long-range approximation to the kick factor works quite well for bunches with $\sigma_{z} / s_{0} \geq 0.5$.

Let us conclude this section by estimating the magnitude of the characteristic distance $s_{0}$. Taking, for instance, a $\mathrm{Cu}$ pipe with $b=1 \mathrm{~cm}$, and using $B$ from Table I we obtain $s_{0} \simeq 30 \mu \mathrm{m}$, which is rather short in comparison to the bunch length values in most accelerators. Thus, in most cases, the simpler long-range formulas of Sec. III should be sufficient. We also note that $c /\left(2 \pi s_{0}\right) \simeq 1.6 \mathrm{THz}$ is less than an order of magnitude from the discussed earlier $14 \mathrm{THz}$ upper frequency limit of the extreme ASE regime for $\mathrm{Cu}$, imposed by Eq. (7). Therefore, at short distances the results of this section should be applied with caution, since it is easy to violate the applicability limits. Finally, it is interesting that, while characteristic distances for extreme ASE and NSE regimes are given by two very different expressions, their values end up being not too different. For instance, for the $b=1 \mathrm{~mm} \mathrm{Cu}$ pipe $s_{0} / s_{0}^{\mathrm{NSE}} \simeq 1.7$, and this ratio monotonically decreases with beam pipe radius reaching 1 for $b \simeq 30 \mathrm{~cm}$.

\section{APPLICATIONS}

Two examples we give below were motivated by early design work on the NSLS-II project, a storage ring light source presently under construction at Brookhaven. The following machine parameters are used for the estimates. We assume $\mathcal{E}=3 \mathrm{GeV}$ electron beam energy, $I_{\mathrm{av}}=$
$500 \mathrm{~mA}$ average beam current, and $\sigma_{z}=4.5 \mathrm{~mm} \mathrm{rms}$ bunch length. The rf frequency is $f_{\mathrm{rf}}=500 \mathrm{MHz}$, harmonic number is 1300 , and the synchrotron frequency is $f_{s}=3.6 \mathrm{kHz}$. We also assume that $80 \%$ of all $\mathrm{rf}$ buckets are uniformly filled and the remaining $20 \%$ of ring circumference is taken up by the ion clearing gap. A more complete list of design parameters is available in [33].

In these examples we consider a superconducting undulator with a $\mathrm{Cu}$-coated vacuum chamber at $\mathrm{LHe}$ temperature. Thus the $\mathrm{Cu}$ layer is in the extreme ASE regime. The chamber has $2 h=5 \mathrm{~mm}$ full vertical gap, and the cross section, not fully defined, has a large horizontal-to-vertical aspect ratio [33]. For $b=h=2.5 \mathrm{~mm}$ the characteristic distance $s_{0} \simeq 13 \mu \mathrm{m} \ll \sigma_{z}$, thus we use the long-range wake formulas of Sec. III. For comparison with room temperature operation we take the $\mathrm{Cu}$ conductivity value of $\sigma_{c}=5.8 \times 10^{7}(\Omega \mathrm{m})^{-1}$.

Instability threshold calculations require total undulator length installed, which is unknown at this time. A conservative estimate in [33] assumed $L=60 \mathrm{~m}$ for the total amount of $5 \mathrm{~mm}$ aperture $\mathrm{Cu}$ pipe in the ring due to smallgap undulators. This is the value we are assuming below for the superconducting undulator example. To concentrate on the ASE resistive wall effects, we are ignoring all other impedance-generating ring components.

Of course, more realistic estimates of instability thresholds must account for other contributions to the total wakefield, such as geometric wake due to various chamber transitions, resistive wall wake due to other accelerator components, etc. An estimate of the contribution of a superconducting undulator to the impedance budget of NSLS-II light source could be found in [33].

\section{A. Resistive wall heat in cold-bore superconducting undulators}

As was mentioned in the Introduction, one of the main design challenges for superconducting undulators is handling potentially substantial heat loads in the cryostat, therefore accurate estimates of beam generated heat are needed [7]. Depending on the detail of the undulator and cryostat design, undulator placement in the accelerator, as well as electron beam parameters, image current heating may or may not dominate over other heat sources such as heat conduction, synchrotron radiation, electron bombardment, etc. Examples for particular machines can be found in [9-11]. The only heat source we are considering below is due to image current.

In this case the average power deposition per unit length of the device is

$$
P / L=\eta f_{\mathrm{rf}} q^{2} k_{\mathrm{loss}} / L=\frac{B Z_{0} c I_{\mathrm{av}}^{2} \Gamma\left(\frac{5}{6}\right)}{4 b \pi^{2} \sigma_{z}^{5 / 3} \eta f_{\mathrm{rf}}},
$$

where $I_{\mathrm{av}}=\eta f_{\mathrm{rf}} q$ is the average current in the ring, $\eta \leq 1$ stands for the fraction of the ring circumference occupied by a bunch train. We assume that all $\mathrm{rf}$ buckets in the train 
are uniformly filled, i.e., bunch spacing equals to the rf wavelength. For other uniform bunch spacings, $f_{\text {rf }}$ should be replaced by the bunch repetition frequency. We will use $\eta=0.8$ for the estimates below.

To approximately account for the noncircular cross section, we relied on the result of Piwinski, see e.g. [3], who showed equal resistive wall losses in the following cases: (1) when a Gaussian beam is centered in a pipe of radius $b$; (2) when it is halfway between infinite plates spaced by $2 b$; and (3) when it is distance $b$ above a single infinite plane. Aiming for a conservative estimate, and allowing for $\Delta=$ $0.5 \mathrm{~mm}$ maximally off-centered beam trajectory we still used Eq. (49) (derived for circular geometry) but put in a reduced value of $b=h-\Delta=2 \mathrm{~mm}$. In this manner the final estimate for NSLS-II parameters came out to $P / L \approx$ $3.2 \mathrm{~W} / \mathrm{m}$.

While fairly significant for cryogenic operation, these power levels were found possible to remove from the undulator cryostat by multiple cryocoolers, see e.g. [7].

For comparison we also estimate power deposition in the same geometry and material for room temperature operation. Keeping the assumptions above, but using the NSE expression for the loss factor, see e.g. [3],

$$
k_{\text {loss }}^{\mathrm{NSE}} / L=\frac{c Z_{0} \Gamma\left(\frac{3}{4}\right)}{4 \pi^{2} b \sigma_{z}^{3 / 2} \sqrt{2 \sigma_{c} Z_{0}}},
$$

we get $P^{\mathrm{NSE}} / L \approx 17 \mathrm{~W} / \mathrm{m}$, i.e., a factor of 5 higher than in the cold case. For in-vacuum permanent magnet undulators operating at room temperature, usually even this modest heat must be managed to avoid thermally induced stress, as well as to minimize detuning phase error due to temperature dependence of magnet remanence.

It is also instructive to compare the result to the losses one would get at LHe temperature disregarding the ASE but using NSE formulas instead. This result would of course scale as $R_{R R}-1 / 2$, and, for instance, for $R R R=$ 100 one gets $1.7 \mathrm{~W} / \mathrm{m}$, which is roughly a factor of 2 lower than the ASE number quoted above.

Finally, we should mention that similar heat load estimates directly from the ASE surface impedance expressions have been done earlier for LHC beam screen studies [5]. That reference, however, did not introduce the loss factor or most other accelerator physics concepts discussed in this paper.

\section{B. Transverse mode-coupling instability}

Because of increasing reliance on small-gap undulators (that present substantial resistive wall impedance as well as geometric impedance due to end tapers) transverse modecoupling instability (TMCI) is becoming more important for modern light sources.

A conservative value for TMCI threshold at zero chromaticity can be found by requiring that the maximum (along the bunch) wakefield-induced betatron tune shift is equal to the synchrotron tune, see e.g. $[1,3,34]$. Since the tune shift is related to the wake potential by

$$
\Delta \nu(z)=-\frac{q W_{\perp}^{\sigma}(z) / L}{4 \pi \mathcal{E} / e} \oint \beta(s) d s
$$

this results in the following threshold:

$$
I_{b}=\frac{4 \pi f_{s} \mathcal{E} / e}{\beta_{0}\left|W_{\perp}^{\sigma}(z)\right|_{\max }},
$$

where $I_{b}$ is the average current per bunch at threshold, $\beta_{0}=\langle\beta(s)\rangle$ is the average value of the beta function along the chamber element creating the transverse wake, and $f_{s}$ is the synchrotron frequency.

To account for the noncircular cross section, we used the results of $[35,36]$ for the resistive wall wake function dependence on the horizontal-to-vertical aspect ratio of a beam pipe with fixed vertical dimension. Increasing the aspect ratio led to a rapid convergence of the vertical and horizontal wake functions to their asymptotic parallel-plate values, equal, respectively, to $\pi^{2} / 12$ and $\pi^{2} / 24$ times $W_{\perp}^{\delta}(z)$ of the round pipe. Based on this we multiplied the round pipe kick factor of Eq. (40) by $\pi^{2} / 12$ [37] to obtain $k_{\text {kick }} / L \simeq 20 \mathrm{~V} / \mathrm{pC} / \mathrm{m}^{2}$. Assuming $L=60 \mathrm{~m}$ for the total length of superconducting undulators installed, we got the value of $\left|W_{\perp}^{\sigma}(z)\right|_{\max } \simeq 1.6 \mathrm{kV} / \mathrm{pC} / \mathrm{m}$. Finally, from Eq. (52) with $\beta_{0}=2 \mathrm{~m}$ [33], we obtained the TMCI threshold of approximately $40 \mathrm{~mA} /$ bunch. This is greater than the nominal operating current of $0.5 \mathrm{~mA} /$ bunch but could be of importance for special modes of operation with higher single bunch current (in selected bunches) and/or shorter bunch length.

For comparison, if these undulators operate at room temperature we can use the NSE expression, e.g. [3],

$$
k_{\text {kick }}^{\mathrm{NSE}} / L=\frac{c Z_{0} \Gamma\left(\frac{1}{4}\right)}{(2 \pi)^{3 / 2} b^{3} \sqrt{\pi \sigma_{c} Z_{0}} \sigma_{z}^{1 / 2}},
$$

and $\left|W_{\perp}^{\sigma N S E}(z)\right|_{\max } \simeq 1.412 k_{\text {kick }}^{\text {NSE }}$ [34], which results in a factor of 4 lower instability threshold, as compared to the ASE case.

TMCI is a single bunch instability. Another instability that is often dominantly driven by the resistive wall impedance is the transverse coupled bunch instability. Its analysis in the regime of extreme anomalous skin effect was given in [38].

\section{CONCLUSION}

We considered resistive wall effects in the regime of the extreme anomalous skin effect. We discussed commonly used accelerator physics concepts involved in collective effect calculations, and derived corresponding mathematical expressions for extreme ASE resistive wall. Since they follow from the ASE surface impedance, these expressions do not depend on metal conductivity $\sigma_{c}$, and they have stronger scaling with distance and/or bunch length as compared to the corresponding expressions for the NSE 
resistive wall. We have also studied the transition to the short range wake regime. It has been shown to occur at the length scale given by the characteristic distance $s_{0}$, introduced in Eq. (43), or, approximately, $s_{0}[\mu \mathrm{m}] \simeq$ $7(b[\mathrm{~mm}])^{3 / 5}$. For beam stay clear apertures of a few $\mathrm{mm}, s_{0}$ is in the tens of $\mu \mathrm{m}$, therefore, except for extremely short bunches, simple long-range expressions of Sec. III are applicable. Two examples we considered at $\sigma_{z}=$ $4.5 \mathrm{~mm}$ rms bunch length, showed a factor of 5 reduction in resistive wall heat load and a factor of 4 increase of (resistive wall dominated) TMCI threshold when switching from the NSE to the extreme ASE regime.

\section{ACKNOWLEDGMENTS}

We wish to acknowledge insightful discussions with G. L. Carr, S. L. Kramer, S. Krinsky, G. Rakowsky, and J.-M. Wang. This work was supported by Department of Energy Contract No. DE-AC02-98CH10886.

[1] A. W. Chao, Physics of Collective Beam Instabilities in High Energy Accelerators (John Wiley \& Sons, Inc., New York, 1993).

[2] B. W. Zotter and S. A. Kheifets, Impedances and Wakes in High-Energy Particle Accelerators (World Scientific, Singapore, 1998).

[3] Handbook of Accelerator Physics and Engineering, edited by A.W. Chao and M. Tigner (World Scientific, Singapore, 1998).

[4] F. Zimmermann, Phys. Rev. E 57, 7146 (1998).

[5] W. Chou and F. Ruggiero, LHC Project Note 2 (SL/AP), 1995.

[6] F. Caspers, M. Morvillo, F. Ruggiero, J. Tan, and H. Tsutsui, in Proceedings of the European Particle Accelerator Conference, Vienna, 2000 (EPS, Geneva, 2000), p. 376.

[7] Proceedings of the Workshop on Superconducting Undulators and Wigglers, ESRF, 2003 (ESRF, Grenoble, 2003), http://www.esrf.eu/Accelerators/Conferences/ IDWorkshop/.

[8] A. Bernhard, M. Hagelstein, B. Kostka, M. Kläser, R. Rossmanith, T. Schneider, M. Weißer, D. Wollmann, E. Steffens, G. Gerlach, and T. Baumbach, IEEE Trans. Appl. Supercond. 16, 1836 (2006).

[9] E. Wallen and G. LeBlanc, Cryogenics 44, 879 (2004).

[10] E. Wallen, J. Chavanne, and P. Elleaume, Nucl. Instrum. Methods Phys. Res., Sect. A 541, 630 (2005).

[11] S. Casalbuoni, A. Grau, M. Hagelstein, R. Rossmanith, F. Zimmermann, B. Kostka, E. Mashkina, E. Steffens, A. Bernhard, D. Wollmann, and T. Baumbach, Phys. Rev. ST Accel. Beams 10, 093202 (2007).

[12] K. L. F. Bane and G. Stupakov, in Proceedings of the 21st Particle Accelerator Conference, Knoxville, 2005 (IEEE, Piscataway, NJ, 2005), p. 3390.

[13] K. L. F. Bane, G. Stupakov, and J. J. Tu, in Proceedings of the 10th European Particle Accelerator Conference,
Edinburgh, Scotland, 2006 (EPS-AG, Edinburgh, Scotland, 2006), p. 2955.

[14] F. Wooten, Optical Properties of Solids (Academic Press, New York, 1972).

[15] S. Lucyszyn, IEE Proc.-Microw. Antennas Propag. 151, 321 (2004).

[16] L. D. Landau, E. Lifshitz, and L.P. Pitaevskii, Electrodynamics of Continuous Media, Landau and Lifshitz Course of Theoretical Physics Vol. 8 (Elsevier, Oxford, 1993), 2nd ed.

[17] C. Kittel, Introduction to Solid State Physics (John Wiley \& Sons, Inc., New York, 2005), 8th ed.

[18] M. Fouaidy and N. Hammoudi, Physica (Amsterdam) 441C, 137 (2006).

[19] P.E. Kirkpatrick, J. W. Ekin, and S. L. Bray, Rev. Sci. Instrum. 70, 3338 (1999).

[20] G. E. H. Reuter and E. H. Sondheimer, Proc. R. Soc. A 195, 336 (1948).

[21] A. B. Pippard, Proc. R. Soc. A 191, 385 (1947).

[22] R. G. Chambers, Proc. R. Soc. A 215, 481 (1952).

[23] D. C. Mattis and J. Bardeen, Phys. Rev. 111, 412 (1958).

[24] M. I. Kaganov, G. Ya. Lyubarskiy, and A. G. Mitina, Phys. Rep. 288, 291 (1997).

[25] E. M. Lifshitz and L.P. Pitaevskii, Physical Kinetics, Landau and Lifshitz Course of Theoretical Physics Vol. 10 (Pergamon, London, 1981).

[26] We are ignoring the difference between the effective mass and the free-electron mass, which may come from band effects, electron-phonon, and electron-electron interaction. The difference should not exceed $20 \%$ in $\mathrm{Cu}$ or $\mathrm{Al}$.

[27] R. B. Dingle, Physica (Amsterdam) 19, 311 (1953).

[28] O. L. Lyubimov, N. M. Makarov, and V.A. Yampolski, Solid State Commun. 39, 815 (1981).

[29] J. T. Rogers, S. De Panfilis, A.C. Melissinos, B.E. Moskowitz, Y.K. Semertzidis, W. Wuensch, H.J. Halama, A. G. Prodell, W. B. Fowler, and F. A. Nezrick, Appl. Phys. Lett. 52, 2266 (1988).

[30] Alternatively one could introduce the wake function as a "generalized function." In this case the inverse Fourier transform of Eq. (22) is well defined and similarly results in Eq. (23).

[31] O. Henry and O. Napoly, Part. Accel. 35, 235 (1991).

[32] K. L. F. Bane and M. Sands, AIP Conf. Proc. 367, 131 (1996); see also K. L. F. Bane and M. Sands, SLAC Report No. SLAC-PUB-95-7074, 1995.

[33] NSLS-II Conceptual Design Report, December 2006, http://www.bnl.gov/nsls2/project/CDR/.

[34] K. Bane and S. Krinsky, in Proceedings of the Particle Accelerator Conference, Washington, DC, 1993 (IEEE, New York, 1993), p. 3375.

[35] K. Yokoya, Part. Accel. 41, 221 (1993).

[36] R. L. Gluckstern, J. van Zeijts, and B. Zotter, Phys. Rev. E 47, 656 (1993).

[37] Our estimate is in the vertical plane since it has a higher product of $k_{\text {kick }} \beta_{0}$. Often the horizontal threshold is lower due to horizontal beta function being more than a factor of 2 larger than the vertical.

[38] B. Podobedov and J.-M. Wang, NSLS-II Technical Note 01, BNL (2003). 